durchgeführt wurde, im Hinblick auf Läsionen der LBS. Eingeschlossen wurden Patienten mit therapierefraktären Schmerzen der LBS nach konservativer Therapie. Die Läsionen wurden nach der anatomischen Lokalisation kategorisiert: innenliegend, junktional und Bizepstunnel. Die innenliegende Läsion waren labrale Risse. Die Läsion, die während einer glenohumeralen Arthroskopie visualisiert werden konnten, wurden als junktionale Läsionen (Pulleyläsion, partielle Rupturen, Synovitis) bezeichnet. Die in der glenohumeral Arthroskopie verborgenen extraartikulären Läsionen der LBS wurden Bizepstunnelläsionen genannt.

\title{
Ergebnisse
}

\section{Schulter-Arthroskopie: Verborgene Läsionen der langen Bizepssehne}

Taylor SA et al. Diagnostic glenohumeral arthroscopy fails to fully evaluate the biceps-labral complex. Arthroscopy 2015; 31: 215-224

Pathologische Veränderungen an der langen Bizepssehne (LBS) treten häufig auf und können chronische Schmerzen im Bereich der Schulter auslösen. Fraglich bleibt weiterhin, wie weit es in einer diagnostischen Arthroskopie der Schulter möglich ist, die Läsionen der langen Bizepssehne, auch extraartikulär, zu detektieren.

\section{Methoden}

Im ersten Schritt wurde bei acht humanen Schulterpräparaten die lange Bizepssehne perkutan während einer diagnostischen glenohumeralen Arthroskopie mittels Fäden markiert. Der prozentuale Anteil der visualisierten LBS wurde relativ zum distalen Rand der Subscapularissehne und dem proximalen Rand der M. pectoralis major berechnet.

Im zweiten Schritt erfolgte eine retrospektive Beurteilung von 277 Patienten, bei denen ein arthroskopischer subdeltoidaler Transfer der LBS auf die conjoint tendon
In den durchführten glenohumeralen $\mathrm{Ar}$ throskopien konnten 78\% der LBS relativ zum distalen Rand der Subscapularissehne und nur 55\% relativ zum proximalen Rand des $M$. pectoralis major visualisiert werden. Der Anteil der LBS inferior der Subscapularissehne wurde nicht sichtbar.

Bei der retrospektiven Analyse stellte sich heraus, dass $47 \%$ der Patienten extraartikuläre Bizepstunnelläsionen hatten. Die häufigsten Pathologien bei diesen Patienten war eine Narbenbildung (48\%) und Instabilität (23\%). Des Weiteren zeigten sich bei 128 Patienten (37\%) Läsionen an multiplen Lokalisationen der LBS. Bei $45 \%$ der Patienten konnte neben einer junktionalen Läsion auch eine Bizepstunnelläsion nachgewiesen werden. Bei 50 Patienten bestand nur eine isolierte Läsion im extraartikulären Bereich der Bizepstunnels.

\section{FAZIT}

Die Studie konnte zeigen, dass eine Standardarthroskopie des Glenohumeralgelenkes nicht zuverlässig alle möglichen Läsionen der LBS nachweisen kann, da ein gewisser Anteil der LBS verborgen bleibt. Des Weiteren ist zu erwähnen, dass ein großer Anteil der Patienten mit Bizepssehnenbeschwerden wahrscheinlich eine $\mathrm{Pa}$ thologie der LBS an mehreren Lokalisationen aufweist.

Dr. med. Marc-Frederic Pastor, Hannover 